\title{
Recombinant factor VIII: past, present and future of treatment of hemophilia $A$
}

\section{S. Raso ${ }^{1,2}$ and C. Hermans ${ }^{2}$}

${ }^{1}$ Azienda Ospedaliera Universitaria Policlinico, Division of Haematology, Department of Surgical, Oncological and Stomatological Disciplines, Palermo, Sicilia, Italy; ${ }^{2}$ Division of Adult Haematology, St-Luc University Hospital, Brussels, Belgium

\section{Contents}

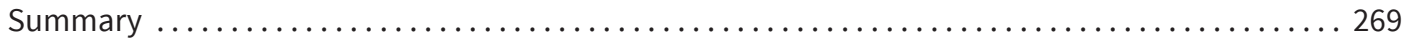

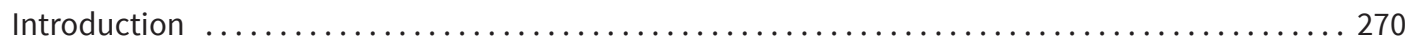

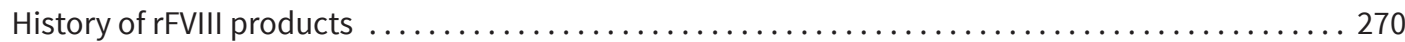

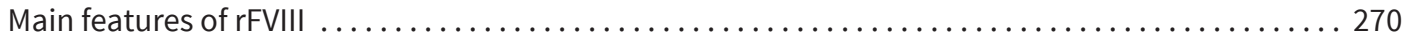

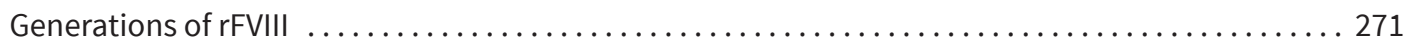

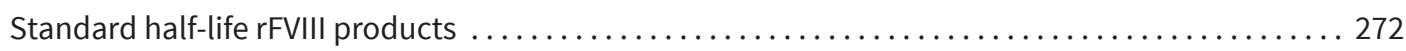

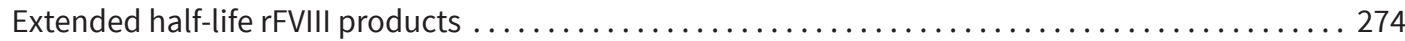

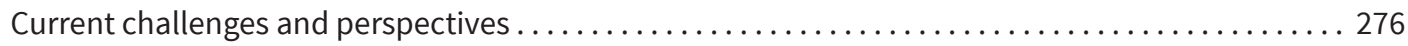

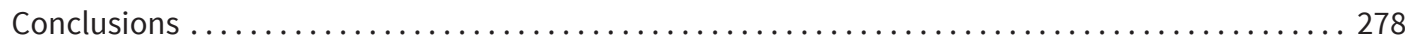

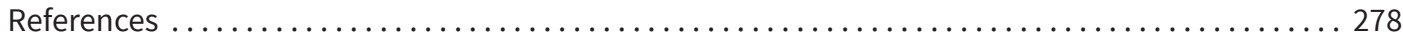

\section{Summary}

The development of recombinant factor VIII (rFVIII) was initially driven by the necessity to treat hemophilia $A$ (HA) patients with FVIII concentrates without the risk of transmitting infectious agents. Over the last three decades the safety of $r F V I I I$ has been further improved by completely removing animal or human proteins from

Correspondence: Cedric Hermans, MD, PhD, FRCP, Division of Adult Haematology, St-Luc University Hospital, Avenue Hippocrate 10, 1200 Brussels, Belgium. E-mail: cedric.hermans@uclouvain.be. the manufacturing process, so that patients would not be exposed to known or emerging pathogens. Recent efforts have concentrated on improving the expression of rFVIII, reducing its immunogenicity and enhancing its pharmacokinetic (PK) behavior. These new goals have been possible thanks to the development of biotechnology and a better knowledge of the function and structure of FVIII. Several approaches such as deletion of the B-domain, expression of FVIII by human cell lines, sequence modification, structural modification, coexpression with other proteins, fusion with the Fc fragment of immunoglobulins 
and PEGylation have been utilized. As a result of these efforts, different rFVIII products have been validated in terms of efficacy, immunogenicity and PK profile. Other technologies are currently being explored to improve the PK of FVIII and allow its subcutaneous administration. Although nonreplacement therapies and HA gene therapy appear to be promising alternatives for HA, rFVIII will very likely remain as a critical component for the treatment of HA because of its physiological activity and mode of action, as well as its unique ability to induce or restore tolerance to exogenous FVIII. This review summarizes the principal features of past, current and emerging rFVIII products for $H A$.

Key words: Hemophilia A - Coagulation factor disorders Recombinant FVIII - Standard half-life FVIII - Extended half-life FVIII

\section{Introduction}

Hemophilia $A(H A)$ and $B$ are congenital X-linked bleeding disorders resulting from a deficiency of clotting factor VIII (FVIII) and IX (FIX), respectively (1). $\mathrm{HA}$ affects 1 in 5,000 male births and it is characterized, according to the basal FVIII level, by spontaneous or traumatic bleeding episodes, which can progress to debilitating arthropathy and significantly impact patients' quality of life (1).

The primary aim of care is to prevent and treat hemorrhagic episodes using replacement therapy with FVIII concentrates, given on demand or prophylactically (2). Recombinant FVIII (rFVIII) products represent a major advance over the past century for the treatment of HA in terms of safety, hemostatic efficacy and manufacturing. The development of neutralizing antibodies, or inhibitors, against exogenous FVIII and the limited half-life ( $\sim 12$ hours) of many available products remain major challenges $(3,4)$.

\section{History of rFVIII Products}

FVIII replacement therapy has been the cornerstone in the treatment of hemophilia and has made major advances over the past century from the administration of blood transfusions to the use of recombinant products in 1992 (5). Prior to the 1950s, whole blood transfusion was the only possible therapy. Subsequently, in the 1960s it was discovered that a fraction cryoprecipitated from plasma contained large amounts of FVIII (6). Purified plasma-derived (pd)FVIII concentrates have been available since the 1970s, and in the following 10 years fractionation and purification processes evolved making plasmaderived concentrates available and allowing home replacement therapy (5).

During the first part of the 1980s, the HIV epidemic devastated the hemophilia community, and in the late 1990 s over $95 \%$ of severe HA patients had been infected by hepatitis C virus through pdFVIII (5). As a consequence, many measures including enhanced donor screening tests and the introduction of viral inactivation have been implemented to improve the infectious safety of plasma-derived concentrates. Nevertheless, the fear of transmitting other unknown, not yet identified and emerging infectious diseases and the desire for safer and more convenient therapies have led to an increasing interest in recombinant DNA technology $(7,8)$.

In 1984 the FVIII gene was cloned and led to the licensure of rFVIII in 1992 (5). rFVIII products, which are derived through heterologous transfection of rFVIII DNA plasmids into a nonhuman mammalian cell line, virtually eliminated blood-borne pathogen transmission (9). The introduction of rFVIII concentrates nearly 20 years ago represented a significant advance in the treatment of HA. The most challenging aspects of HA management today are the occurrence of FVIII inhibitors and the burden of frequent intravenous infusions required for prophylactic replacement therapy (5).

\section{Main Features of rFVIII}

The human FVIII (hFVIII) gene is localized on the long arm of the $\mathrm{X}$ chromosome and its expression is predominantly in sinusoidal and vascular endothelial cells in the liver and the lungs $(10,11)$. After translation, the protein undergoes further processing of $\mathrm{N}$-linked glycans, sulfation of specific tyrosine residues, copper binding, O-linked glycosylation and intracellular proteolysis within the Golgi apparatus and endoplasmic reticulum prior to secretion into plasma $(12,13)$. The mature FVIII is a complex 300-kD heterodimeric glycoprotein consisting of six structural domains and three acidic subdomains, 
organized in a heavy chain $[\mathrm{A} 1(\mathrm{a} 1) \mathrm{A} 2(\mathrm{a} 2) \mathrm{B}]$ and a light chain [(a3)A3C1C2] (12).

rFVIII is one of the largest and most complex proteins manufactured commercially to date (14). Because of its structure and post-translational modification requirements, production of $\mathrm{rFVIII}$ depends on the use of mammalian cell lines, and either Chinese hamster ovary $(\mathrm{CHO})$ or baby hamster kidney (BHK) cells were initially used. In fact, both the $\mathrm{CHO}$ and BHK cell lines have demonstrated their capacity to synthesize rFVIII that includes all of the posttranslational modifications necessary for its hemostatic activity $(9,14)$.

The expressed proteins are secreted into a culture medium, resulting in a large-scale production of rFVIII products, which in some cases contain human and animal proteins for stabilization $(15,16)$. The purification process of rFVIII involves many steps, such as multiple chromatography columns, including immunoaffinity and cation exchange (17), followed by a solvent/detergent step, and either ultra- or nanofiltration to reduce transmission of enveloped (i.e., HIV, hepatitis B and hepatitis C virus) and nonenveloped viruses (i.e., hepatitis $A$ virus and parvovirus B19) (9).

Conventional rFVIII concentrates behave similarly to pdFVIII with regard to biochemical and hemostatic properties. In fact, both proteins have similar pharmacokinetic (PK) profiles and cleavage patterns in vitro (18). However, minor differences in the post-translational modifications are observed in FVIII molecules derived from different species and cells lines (19). For example, carbohydrate structural differences specific to the expression cell lines are present; for instance $\mathrm{CHO}$ cell lines can synthesize the glycan epitope $\mathrm{N}$-glycolyl neuraminic acid (Neu5Gc) moiety, which is not present in the endogenous FVIIIs (13). Similarly, BHK cells synthesize Gal $\alpha 1 \rightarrow 3 \mathrm{Gal}$ groups onto some of their polyantennary sugar chains, which are not normally present in human or primate glycoproteins (19). The expression of Gal $\alpha 1 \rightarrow 3 \mathrm{Gal}$ groups has been suggested to be a potential source of immunogenicity. These cell lines have been the only source of rFVIII products with good clotting efficacy and excellent viral safety for more than 30 years $(13,15)$. However, newer products have been recently made using human embryonic kidney (HEK) cells (13). This HEK-derived rFVIII product (human-cl rhFVIII) does not contain Gal $1 \rightarrow 3$ Gal and Neu5Gc epitope (19).

Sulfation is another post-translational modification important for the function of FVIII (20). Six active sulfation sites of tyrosine residues have been identified in hFVIII and all six sulfation sites are necessary for the full procoagulant activity of FVIII. Sulfation of the residue Y1680 (Tyr 1680) is considered crucial for the stability of FVIII and its binding to von Willebrand factor (VWF) (13). The proportion of Tyr 1680 is high in BHK-derived rFVIII products as well as in other rFVIII products made from different cell lines, ranging from $1 \%$ to $6.5 \%$ for second-generation rFVIII to up to $15 \%$ for third-generation rFVIII (21).

\section{Generations of rFVIII}

Several preparations of rFVIII are currently available for patients with HA (5). Manufacturing of rFVIII evolved over decades and provided products that were classified depending on whether animalderived or human-derived proteins were used during production and were present in the final formulation (22).

rFVIII products can be divided in the following three different generations: i) first-generation products using animal-derived proteins in the cell culture medium and human serum albumin in the final formulation to stabilize FVIII; ii) second-generation products using human-derived proteins in the culture medium but with no albumin added in the final formulation; and iii) third-generation products manufactured without addition of animal or human proteins during processing or in the final formulation (21) (Table I).

First-generation rFVIII products (Kogenate; Recombinate) contain both animal- and human-derived proteins in the cell culture as well as human albumin added in the final formulation as stabilizers (21). To improve safety, second-generation rFVIII concentrates (Kogenate FS; Helixate FS) have removed human albumin and have used sucrose as a stabilizing agent since 2000. Moreover, the human serum albumin used in the cell culture process has been removed prior to the final formulation. Third-generation products (Advate; ReFacto), which have been licensed 
Table I. Characteristics of first developed recombinant FVIII products.

\begin{tabular}{|c|c|c|c|c|c|c|}
\hline Generation & $\begin{array}{l}\text { Product } \\
\text { (manufacturer) }\end{array}$ & FVIII & Cell line & Culture medium & Stabilizer & $\begin{array}{c}\text { Purification/viral } \\
\text { inactivation }\end{array}$ \\
\hline First & $\begin{array}{l}\text { Recombinate } \\
\text { (Shire) }\end{array}$ & Full-length & $\mathrm{CHO}$ & $\begin{array}{l}\text { Bovine serum } \\
\text { albumin }\end{array}$ & Human albumin & IAC/IEC \\
\hline Second & $\begin{array}{l}\text { Kogenate FS } \\
\text { (Bayer) }\end{array}$ & Full-length & $\mathrm{BHK}$ & $\begin{array}{l}\text { Human plasma } \\
\text { protein solution }\end{array}$ & Sucrose & $\mathrm{IAC} / \mathrm{IEC} / \mathrm{SD} / \mathrm{UF}$ \\
\hline Second & $\begin{array}{l}\text { Helixate FS } \\
\text { (CSL Behring) }\end{array}$ & Full-length & $\mathrm{BHK}$ & $\begin{array}{l}\text { Human plasma } \\
\text { protein solution }\end{array}$ & Sucrose & $\mathrm{IAC} / \mathrm{IEC} / \mathrm{SD} / \mathrm{UF}$ \\
\hline Third & $\begin{array}{l}\text { Advate } \\
\text { (Shire) }\end{array}$ & Full-length & $\mathrm{CHO}$ & None & Trehalose & $\mathrm{IAC} / \mathrm{IEC} / \mathrm{SD}$ \\
\hline Third & $\begin{array}{l}\text { ReFacto } \\
\text { (Pfizer) }\end{array}$ & $\begin{array}{l}\text { B-domain } \\
\text { deleted }\end{array}$ & $\mathrm{CHO}$ & None & Sucrose & $\mathrm{IAC} / \mathrm{IEC} / \mathrm{SD} / \mathrm{NF}$ \\
\hline
\end{tabular}

BHK, baby hamster kidney; CHO, Chinese hamster ovary; FVIII, factor VIII; IAC, immunoaffinity chromatography; IEC, ion exchange chromatography; NF, nanofiltration; SD, solvent/detergent treatment; UF, ultrafiltration.

since 2003, do not use any human or animal proteins in the cell culture or the production of FVIII products $(21,22)$.

rFVIII concentrates can also be divided into full-length (FL) or B-domain-deleted (BDD) concentrates, in which the B-domain is removed from the FVIII molecule (21). Early on in the study of rFVIII expression, it was demonstrated that the B-domain of FVIII could be removed without loss of FVIII final clotting efficacy (18). This modification significantly improved secretion from the cell in the recombinant process, and thus BDD-FVIII products such as ReFacto were developed (23). In order to show the efficacy of BDDFVIII, direct comparison studies were performed using BBD-FVIII (ReFacto) and FL-rFVIII (Advate). The direct PK comparisons showed that the FVIII products were bioequivalent to each other (24).

Conventional rFVIII concentrates differ in protein sequence or manufacturing process but offer patients similar PK and security profiles (17). Clinical studies have shown that the recombinant coagulation factors provide safe, well-tolerated and effective treatment of HA whether used in on-demand therapy, prophylactic regimens or surgery (15). To date, there is no consensus regarding whether any one of the currently available rFVIII products has significant clinical advantage over the others. Each product has a strong safety record and has been available for a number of years in many countries. Moreover, there are many studies in patients with HA but randomized clinical trials comparing one generation with another product or generation have not been conducted and are unlikely to take place in the future (25).

\section{Standard Half-life rFVIII Products}

Since 2013, the U.S. Food and Drug Administration (FDA) has licensed four additional third-generation rFVIII concentrates with standard half-life (17). Each of these products has been distinguished from other available factor concentrates by changes in cell line, protein structure or manufacturing techniques (Table II). The first of these new rFVIII products to be approved by the U.S. FDA is turoctocog alfa (NovoEight), a B-domain truncated rFVIII produced in $\mathrm{CHO}$ cell lines without addition of any human- or animal-derived materials. Its primary structure (called N8 protein) contains a B-domain that is truncated to 10 amino acids from the $\mathrm{N}$-terminus and 11 amino acids from the $\mathrm{C}$-terminus, and shows sulfation and glycosylation patterns very similar to those of pdFVIII (26). Glycosylation of N8, as for the pdFVIII, is mainly characterized by the presence of four $\mathrm{N}$-linked glycosylations and one O-linked glycosylation (Ser750) in the B-domain $(26,27)$. Moreover, nonhuman glycan epitopes, critically expressed by different culture cells, are absent (16). As mentioned above, it has been hypothesized that these nonhuman epitopes carry potential implications for immunogenicity (13).

Two phase III trials showed the safety and efficacy of turoctocog alfa in previously treated patients (PTPs) 
Table II. Characteristics of new standard half-life recombinant FVIII products.

\begin{tabular}{|c|c|c|c|c|c|}
\hline $\begin{array}{l}\text { Brand name } \\
\text { (manufacturer) }\end{array}$ & Generic name & $\begin{array}{l}\text { Frequency of i.v. } \\
\text { administration }\end{array}$ & $\begin{array}{l}\text { Mean ABR } \\
\quad(95 \% \mathrm{Cl} \text { or } \pm \mathrm{SD})\end{array}$ & $\begin{array}{l}\text { Median ABR } \\
\text { (IQR or range) }\end{array}$ & References \\
\hline $\begin{array}{l}\text { NovoEight } \\
\text { (Novo Nordisk) }\end{array}$ & Turoctocog alfa & $3 \times$ weekly or q2d & $6.5(5.3-8.0)$ & $3.7(8.7)$ & $(28-31)$ \\
\hline $\begin{array}{l}\text { Nuwiq } \\
\text { (Octapharma) }\end{array}$ & Simoctocog alfa & $3 \times$ weekly or q2d & $\begin{array}{l}2.3( \pm 3.7)^{\mathrm{a}} \\
4.1( \pm 5.2)^{\mathrm{b}}\end{array}$ & $\begin{array}{l}0.9(0-14.7)^{\mathrm{a}} \\
1.9(0-20.7)^{\mathrm{b}}\end{array}$ & $(42,43)$ \\
\hline $\begin{array}{l}\text { Kovaltry } \\
\text { (Bayer) }\end{array}$ & Octocog alfa & $\begin{array}{l}2 \times \text { weekly } \\
3 \times \text { weekly } \\
2 \times \text { weekly } \\
3 \times \text { weekly }\end{array}$ & $\begin{array}{l}3.8( \pm 5.2) \\
4.9( \pm 6.8)\end{array}$ & $\begin{array}{l}1(0.0-8) \\
2(0.5-5) \\
4(0.0-8) \\
2(0.0-4.9)\end{array}$ & $(32-34)$ \\
\hline $\begin{array}{l}\text { Afstyla } \\
\text { (CSL Behring) }\end{array}$ & Lonoctocog alfa & 2-3 $\times$ weekly & $3.1( \pm 5.1)$ & $1.14(0.0-4.2)$ & $(38-40)$ \\
\hline
\end{tabular}

adult patients.

bediatric patients.

IQR, difference between $75^{\text {th }}$ percentile ( $3^{\text {rd }}$ quartile) and $25^{\text {th }}$ percentile ( $1^{\text {st }}$ quartile); $\mathrm{ABR}$, annual bleeding rate; $\mathrm{Cl}$, confidence interval; SD, standard deviation; q2d, every 2 days.

in the prevention and treatment of hemorrhagic episodes: Guardian 1 in adult/adolescent patients and Guardian ${ }^{\mathrm{TM}} 3$ in pediatric patients $(28,29)$. Interim results from Guardian 2 showed a favorable long-term effect of turoctocog alfa on annualized bleeding ratio $(A B R)(30,31)$. No FVIII inhibitors were detected. The Guardian clinical trial program is still ongoing with Guardian 4 (ClinicalTrials.gov Identifier NCT01493778), a phase III trial in pediatric previously untreated patients (PUPs) with HA (30).

In 2016, octocog alfa (BAY-81-8973, Kovaltry) was FDA-approved on the basis of the "Long-Term Efficacy Open-Label Program in Severe Hemophilia A Disease" (LEOPOLD) clinical trial program LEOPOLD I (32), LEOPOLD II (33) and Kids trials (34). It is an unmodified FL-rFVIII produced in BHK cell lines with the same amino acid sequence as its predecessor (Kogenate FS), but with advanced manufacturing technologies. The changes in its production consist in coexpression of heat shock protein 70 (HSP70) in the new cell bank and production without addition of human- or animal-derived materials to the cell culture, purification or formulation processes (35). These changes led to differences in sialylation of the rFVIII product that is characterized by a high degree of sialic acid capping of $\mathrm{N}$-terminal glycans on the molecular surface. This new sialylation profile is believed to be responsible for the $10 \%$ longer half-life of Kovaltry when compared with Kogenate FS in crossover PK studies (35). Kovaltry showed a low ABR when used as prophylaxis in PTPS (adult and pediatric) with severe HA $(33,34)$. Infusion of the product was generally well tolerated, with no inhibitor development reported in PTPs.

Lonoctocog alfa (rVIII-SingleChain, CSL-627, Afstyla) is the only rFVIII with a specific single-chain design which increases its stability during manufacturing and displays a stronger affinity for VWF (36). It is produced in $\mathrm{CHO}$ cells without the use of human- or animalderived proteins, and purified using chromatographic techniques (37). The characteristic design of this new molecule consists of a single chain in which the heavy and light chains are covalently liked by a truncated B-domain. This structure differs from other rFVIII preparations, as these have two-chain molecules that are linked via a labile metal-ion bridge (36). Even if VWF exerts a protective effect on FVIII from degradation and clearance, the faster and tighter binding of lonoctocog alfa to VWF determines only a slight prolongation of its half-life when compared to octocog alfa (14.5 vs. 13.3 hours) $(38,39)$. It is to be noted that one-stage assays (OSAs) underestimate FVIII procoagulant activity (FVIII:C) levels in patients receiving lonoctocog alfa by approximately $45-50 \%$, whereas chromogenic substrate assays (CSAs) are closer to target. The underestimation affecting the calculation of FVIII activity with lonoctocog alfa in OSAs could be predictable and corrected by multiplying the results by a conversion factor of 2 (36). Safety and clinical efficacy of the molecule were demonstrated in the AFFINITY program, which consists of 
two pivotal studies (one with an adult/adolescent population and the other with a pediatric population, both completed), as well as an ongoing extension study (subjects 65 years or younger) to assess longterm safety data (40).

Finally, simoctocog alfa (human-cl rhFVIII, Nuwiq) is the first BDD-rFVIII produced in HEK cell lines. It was approved by the FDA in 2015. Manufacturing of HEKderived products like simoctocog alfa, as mentioned above, ensured a human pattern of post-translational modifications (41). Its post-translational modifications (full sulfation of Tyr 1680) increased the VWFbinding affinity (16). The impact of post-translational modifications on immunogenicity is being investigated in the NuProtect study (GENA-05, ClinicalTrials. gov Identifier NCT01992549), which is still ongoing. Preliminary data results from an interim analysis demonstrated low inhibitor rates, with the cumulative incidence being 20.8\% (95\% confidence interval [Cl], 10.68-30.95) for all inhibitors and 12.8\% (95\% $\mathrm{Cl}, 4.49-21.25)$ for high-titer inhibitors (42). Several phase I/II and III trials have demonstrated the safety and efficacy of simoctocog alfa in the prevention and treatment of bleeds in PTPs with severe HA (43).

\section{Extended Half-life rFVIII Products}

Over the last 30 years the main goal in developing new treatments for hemophilia has been to increase the safety of concentrates with respect to viral and other infectious agent transmissions. Recently, due to the improvements in safety obtained by recombinant manufacturing of FVIII, the attention has shifted to modifying the FVIII molecule to improve replacement therapy. In particular, attempts were made to obtain FVIII products with extended halflife (EHL-FVIII) and enhanced functional activity, and to decrease immunogenicity (44). Researchers have assessed several protein modification techniques to obtain FVIII products with a prolonged half-life with the purpose of supporting an extension of the treatment intervals required for effective prophylaxis or higher trough levels with a standard number of infusions (17).

Among these newer techniques, to date only two approaches have led to regulatory approval: i) covalent attachment of FVIII to polyethylene glycol (PEG; PEGylation) and ii) fusion to the Fc portion of the
IgG1 molecule (45). An alternative approach is modification with polysialic acid technology (17), which leads to interference of the receptor-mediated clearance (46). BAX-826 (Shire) is a polysialylated FL-rFVIII that was in phase I clinical trials for the treatment of HA (17), but its development was discontinued by Shire as the principal objective in the clinical trials was not achieved. So far, fusion of FVIII to albumin has been unsuccessful in preserving effective coagulation activity.

The findings obtained with the current EHL-FVIII products have been marked by a limited expansion of their half-life that is only 1.5- to 1.7-fold longer than that of wild-type factor (44) (Table III). On the other hand, the current status of FIX long-acting products is at the moment more attractive since the prolongation of their half-life is as great as 4- to 5 -fold (44).

\section{PEGylation}

PEGylation consists of a covalent link between PEG (a hydrophilic inert substance) and a protein. The molecular weight and the size of the PEGylated protein are increased leading to reduced renal elimination, providing decreasing receptor-mediated clearance and steric shield against immunological recognition (46). While questions have been raised about retention of PEG in the reticuloendothelial system, the safety of PEG and licensed therapeutics using PEG technology has been demonstrated in numerous studies $(17,47)$. The method of PEGylation varies by manufacturer. Of the several PEG-FVIII molecules that have been created only three EHL products have reached clinical trials (Table III). An explanation for this phenomenon is the interference of PEG with the clotting activity of FVIII by altering its interaction with other molecules, including VWF (48). It has been hypothesized that PEGylation could have a beneficial impact on immunogenicity by interfering with the interaction of FVIII with antigen-presenting cells.

Rurioctocog alfa pegol (BAX-855, Adynovate) contains the native FL-rFVIII protein used in Advate coupled with 20-kDa branched PEG molecules (47). It was approved by the FDA in November 2015. Rurioctocog alfa pegol is prepared using a proprietary stable PEGylation technology developed by Nektar Therapeutics which is targeted at the 
Table III. Characteristics of extended half-life FVIII products.

\begin{tabular}{|c|c|c|c|c|c|c|c|}
\hline Product & $\begin{array}{c}\text { Approval } \\
\text { year }\end{array}$ & Modification & Cell line & $\begin{array}{l}\text { Half-life } \\
\text { (hours) }\end{array}$ & $\begin{array}{l}\text { Prophylaxis } \\
\text { regimen }\end{array}$ & Manufacturer & References \\
\hline $\begin{array}{l}\text { Efmoroctocog } \\
\text { alfa } \\
\text { (Eloctate) }\end{array}$ & 2014 & $\begin{array}{l}\text { BDD-rFVIII } \\
\text { with fused Fc }\end{array}$ & HEK & 19 & $\begin{array}{l}\text { 25-65 IU/kg } \\
\text { every } \\
3 \text { to } 5 \text { days }\end{array}$ & Biogen/Sobi & $(56-61)$ \\
\hline $\begin{array}{l}\text { Rurioctocog } \\
\text { alfa pegol } \\
\text { (Adynovate) }\end{array}$ & 2015 & $\begin{array}{l}\text { Full-length rFVIII } \\
\text { with 20-kDa } \\
\text { nonspecific } \\
\text { PEGylation }\end{array}$ & $\mathrm{CHO}$ & $14-19.6$ & $\begin{array}{l}40-50 \mathrm{IU} / \mathrm{kg} \\
2 \times \text { weekly }\end{array}$ & Shire & $(47,49,50)$ \\
\hline $\begin{array}{l}\text { Turoctocog } \\
\text { alfa pegol } \\
\text { (N8-GP) }\end{array}$ & - & $\begin{array}{l}\text { B-domain truncated } \\
\text { rFVIII with 40-kDa } \\
\text { site-specific } \\
\text { PEGylation }\end{array}$ & $\mathrm{CHO}$ & 19 & $\begin{array}{l}50 \mathrm{IU} / \mathrm{kg} \text { every } \\
4 \text { days }\end{array}$ & Novo Nordisk & $(48,50,53,54)$ \\
\hline $\begin{array}{l}\text { Damoctocog } \\
\text { alfa pegol } \\
\text { (Bay-94-9027) }\end{array}$ & - & $\begin{array}{l}\text { BDD-rFVIII with } \\
\text { 60-kDa } \\
\text { site-specific } \\
\text { PEGylation }\end{array}$ & BHK & 18.7 & $\begin{array}{l}\text { 30-40 IU/kg } \\
\text { twice weekly } \\
\text { or } 45-60 \mathrm{IU} / \mathrm{kg} \\
\text { every } 5 \text { days }\end{array}$ & Bayer & $(51,52)$ \\
\hline
\end{tabular}

rFVIII, recombinant factor VIII; Fc, fragment crystallizable; BDD, B-domain deleted; CHO, Chinese hamster ovary; HEK, human embryonic kidney; BHK, baby hamster kidney.

$\varepsilon$-amino groups of lysines (49). The manufacturers utilized techniques to remove the excess of free PEG reagent, as well as highly PEGylated and non-PEGylated $\mathrm{rFVIII}$, and produced approximately 2 mol of PEG per FVIII molecule to enhance the PK profile of rFVIII (49). Two clinical studies were conducted in male patients with severe HA to assess the safety, efficacy, PK profile and immunogenicity of rurioctocog alfa pegol administered as prophylactic and on-demand treatments. Subjects on prophylaxis (40-50 IU/kg twice weekly) had a significantly reduced (90\%) ABR compared with those following the on-demand treatment. The half-life of rurioctocog alfa pegol was found to be 1.4-fold longer (1419.6 hours) than the half-life of Advate. No incidence of inhibitor development was described and no significant reagent-dependent differences between assays were seen (50).

Damoctocog alfa pegol (BAY-94-9027) is a B-domain truncated rFVIII variant that is site-specifically conjugated with a 60-kDa PEG linked via an amino acid residue substitution by cysteine. In a phase I trial, damoctocog alfa pegol was compared to rFVIII Kogenate FS and showed a half-life of about 19 hours (51). In the PROTECT VIII, phase II/III study, damoctocog alfa pegol showed a median ABR of 1.9 and 3.9 when given as prophylaxis every 5 (45-60 IU/kg) and 7 (60 IU/kg) days, respectively, in PTPs with severe HA (52). During the studies, the new EHL-FVIII was well tolerated without inhibitor development. In patients that receive damoctocog alfa pegol the FVIII:C can be measured using CSAs and OSAs that use an ellagic acid-based reagent (50).

Another glycoPEGylated agent, not yet approved for use, is turoctocog alfa pegol (N8-GP). Turoctocog alfa pegol corresponds to B-domain truncated rFVIII (turoctocog alfa) glycoPEGylated with a 40-kDa PEG that is attached via enzymatic transfer to a unique O-linked glycan in the 21-amino acid residual B-domain. It exhibited a mean half-life of 19.0 hours (range 11.6-27.3 hours), which represented approximately a 1.6-fold prolongation in comparison with rFVIII products previously used by the patients (53). With turoctocog alfa pegol the FVIII:C in OSAs varied with different activated partial thromboplastin time reagents leading to a significant underestimation (50). In clinical studies, turoctocog alfa pegol was in general well tolerated and a low-titer inhibitory antiFVIII antibody was reported in only 1 patient after 93 days of exposure (54). The results of PATHFINDER 2, a phase III study in PTPs (adolescent and adult), showed a median ABR of 1.3 and 30.9 for prophylactic (50 IU/kg every 4 days) and on-demand treatment, respectively (53). 


\section{Fc fusion}

The neonatal Fc receptor (FcRn) protects IgG and albumin from lysosomal breakdown and is involved in antigen presentation by professional antigenpresenting cells (44). The capacity of this receptor to extend the half-life of IgG and albumin has led to the development of new treatments (55). Fc fusion was the first half-life extension technology to be used in the hemophilia field. Fusion of the monomeric form of the IgG constant region (Fc) allows binding to the neonatal FcRn, delaying lysosomal degradation of Fc-containing proteins by cycling them back into circulation. The FVIII-Fc fusion protein induces half-life extension by altering the normal clearance (56).

Efmoroctocog alfa (rFVIIIFc, Eloctate) is a molecule in which Fc (from human IgG1) is linked to the C-terminus of BDD-rFVIII and is expressed in HEK293H cells (57). The phase III A-LONG study demonstrated an extended half-life of rFVIIIFc as well as its safety and hemostatic efficacy, including surgical prophylaxis, in previously treated adults/adolescents and children with severe HA (58-60). Results following tailored prophylaxis with efmoroctocog alfa (25$65 \mathrm{lU} / \mathrm{kg}$ every 3 to 5 days), according to individual patients' PK, revealed a median ABR of 1.6 (59).

Subsequently, interim data from the ASPIRE extension study confirmed the maintenance of low ABRs with extended prophylactic dosing intervals in patients receiving efmoroctocog alfa prophylaxis (61). The mean half-life of rFVIIIFc was found to be 19 hours and increased approximately 1.5 -fold relative to that of standard rFVIII. This prolongation of half-life is a modest improvement compared to the 5-fold half-life extension achieved with rFIXFc (44). This limited improvement seems to be in part related to the close interaction of FVIII with VWF, its carrier protein in the circulatory system (62). It is possible to assess the plasma levels of FVIII activity after use of rFVIIFc by CSAs and OSAs, although both can vary significantly by reagent (50). A phase III trial (ClinicalTrials.gov Identifier NCT02234323) in PUPs with severe $\mathrm{HA}$ is enrolling participants to evaluate the safety and efficacy of rFVIIIFc.

\section{Recombinant porcine FVIII}

The sequence differences between hFVIII and porcine FVIII ( $p F V I I I)$ result in lower cross-reactivity of
anti-hFVIII alloantibodies with pFVIII (63). For this reason $\mathrm{pFVIII,} \mathrm{derived} \mathrm{from} \mathrm{plasma,} \mathrm{was} \mathrm{used} \mathrm{since}$ the 1960s until 2004, when its manufacturing ceased due to concerns related to its safety and side effects (hypersensitivity and thrombocytopenia) (63). Susoctocog alfa (OBI-1, Obizur), a BDD recombinant pFVIII ( $r$-pFVIII), has been produced in BHK cell lines and manufactured using two viral clearance steps to reduce the risk of potential pathogen transmission (64).

On the basis of the results of a prospective, multicenter, phase II/III study that evaluated the efficacy and safety of susoctocog alfa for the treatment of serious hemorrhagic events in patients with acquired HA (AHA), it was approved by the FDA as therapy for bleeds in patients with AHA. In the study, which included 28 patients with AHA, bleeds were successfully controlled with r-pFVIII in $86 \%(24 / 28)$ of subjects (64). Meanwhile, a phase II study assessing treatment of non-life/non-limb-threatening bleeding in patients with congenital HA and FVIII inhibitors showed that administration of eight or fewer injections of r-pFVIII resulted in hemostasis in 25 bleeding events (65). Patients tolerated r-pFVIII well without significant adverse events. A trial evaluating the use of $r$-pFVIII in patients with congenital HA and inhibitors undergoing surgery is currently ongoing (ClinicalTrials.gov Identifier NCT02895945).

\section{Current Challenges and Perspectives}

rFVIII was initially developed and produced in response to the need of a safe treatment, in terms of infection transmission, after the devastating sequelae of the AIDS and hepatitis epidemics of the 1980 s in the hemophilia community. Since the risk that recombinant products can contain infectious contaminants is minimal, as there is a low probability that infectious agents may enter the cell culture and manufacturing processes, rFVIII products have largely eliminated concerns regarding pathogen transmission $(21,66)$.

Since 1988, the use of recombinant products has progressively increased and in many industrialized countries the plasma-derived products have nearly been completely replaced. Correspondingly, the worldwide demand of recombinant products raised up from 1.3 billion units in 1984 to 5.5 billion units 
in 2008 (66). The growing availability and quality of recombinant coagulation factors have improved the quality of life of children with hemophilia and their families, have ensured home treatment and have contributed to the broad implementation of prophylactic treatment regimens in both adults and children $(7,66)$.

The major adverse consequence of using FVIII concentrates is the development of neutralizing inhibitors, which occur in about $30 \%$ of patients with severe $\mathrm{HA}$ and thus reduce or annul the response to treatment (67). The pathogenesis of inhibitory anti-FVIII antibodies is a multifactorial process that involves immune regulatory molecules, whose action and level are both genetically and nongenetically defined, and environmental factors. Nongenetic risk factors include those which are treatment-related, such as dosing regimen and type of product (68).

In the past, it was often suggested that there was a lower incidence of FVIII inhibitors with treatment based on plasma-derived concentrates than on recombinant concentrates. However, the studies performed to date have reported conflicting results (68), underlining the immunogenic role of infused FVIII, whether plasma-derived or recombinant. Only recently, the SIPPET study, a prospective randomized trial assessing the incidence of FVIII inhibitors, has reported a significantly increased risk of inhibitor development in PUPs treated with rFVIII compared with pdFVIII, raising the debate regarding factor concentrate-related immunogenicity (69).

Recently, rFVIII concentrates have been produced employing technological strategies to reduce FVIII immunogenicity (i.e., use of human cell lines, higher affinity towards VWF, no human or animal impurities) $(36,41)$. Moreover, results from preclinical studies support the notion that the Fc protein of rFVIIIFc has immunomodulatory properties $(70,71)$. However, the supposed lower immunogenic profile of these newer rFVIII concentrates needs confirmation in further studies including PUPs.

Even if the latest generations of rFVIII concentrates have led to some improvements, at the moment they have not eliminated the risk of inhibitor development. Therefore, in order to overcome the limitations of replacement therapy, there are emerging technologies that attempt to restore hemostasis outside
FVIII/FIX replacement by using bioengineered substitutes that enhance thrombin generation or by inhibiting the natural anticoagulants (tissue factor pathway inhibitor, antithrombin and protein C) (72).

New nonfactor replacement therapies include a single molecule named emicizumab, which is a humanized, bispecific monoclonal antibody targeting FIXa and FX that can function as a FVIIIa mimetic. The bispecific monoclonal antibody emicizumab replaces only part of the full FVIIIa cofactor activity, and since it is a different protein, it differs greatly from FVIIla in terms of affinity, regulation and typology, as well as regarding FIXa-enhancing activity (73).

Other nonfactor replacement therapies are i) concizumab, a humanized monoclonal IgG4 antibody that arrests the action of tissue factor pathway inhibitor; ii) fitusiran, a short-interfering RNA therapy that reduces antithrombin function in a dose-dependent way (74); and iii) activated protein C-specific serpin or protein S silencing RNA (75).

While concizumab and fitusiran are being evaluated in phase II/III trials, emicizumab has obtained FDA approval for the treatment of patients with $\mathrm{HA}$ and FVIII inhibitors (74). In fact, results from the HAVEN 1 and 2 studies showed that emicizumab prophylaxis was associated with a significantly lower rate of bleeding events in this setting of patients. It exhibits a long half-life (several weeks) and is administered via subcutaneous route, thus representing an attractive and significant change for hemophilia care (74).

These innovative strategies can be used for prophylaxis therapy, but some breakthrough bleeds are inevitable and these may require additional hemostatic treatment. The best choice of additional treatment in patients without inhibitors is represented by FVIII products, and by bypassing agents in the case of patients with inhibitors $(76,77)$. However, the need to treat breakthrough bleeding and avoid repeated high doses of bypassing agents requires the restoration of tolerance to FVIII in patients with inhibitors $(76,77)$.

The only proven effective therapy for inhibitor eradication is Immune Tolerance Induction (ITI), which involves repeated and persistent injections of replacement factor in order to downregulate the established antibody response and induce immune 
tolerance. For the patients in whom ITI fails, options are few and are not clearly effective (72). Regarding type of concentrate, the choice of FVIII product to obtain inhibitor eradication is also a matter of debate (78). Even if there is no current information to suggest a more favorable outcome for one product over another, international consensus guidelines state that the majority of patients are tolerized with the same FVIII product that was in use at the time of inhibitor detection. This is a successful approach, and no evidence exists that supports switching to a different FVIII concentrate for de novo ITI. Otherwise FVIII concentrates containing VWF are currently recommended for salvage ITI in patients who have failed previous attempts using monoclonal or rFVIII products (79).

As summarized above, several different rFVIII concentrates, each with specific features, have been extensively validated in terms of hemostatic efficacy, immunogenicity and kinetic behavior, and are available for the treatment of patients with HA. However, in spite of these developments, replacement therapy with high-tech rFVIII remains problematic in many patients. Other technologies are currently being explored to allow, by additional modifications, subcutaneous administration of rFVIII and to significantly increase its half-life in blood. For instance, the $\mathrm{PK}$, effectiveness and local distribution of subcutaneously administered turoctocog alfa pegol were recently evaluated in preclinical models; PK modeling predicted FVIII trough levels of $2.5-10 \%$ with turoctocog alfa pegol administered subcutaneously at a daily dose of $12.5 \mathrm{IU} / \mathrm{kg}^{-1}$ in $95 \%$ of patients with severe $\mathrm{HA}(80)$.

\section{Conclusions}

Although a cure for hemophilia has recently been achieved in some patients by gene therapy $(81,82)$, in the last 20 years the production of rFVIII has revolutionized the treatment of hemophilia in terms of security, efficacy and treatment burden. The availability of a wide choice of safe and increasingly effective concentrates, including newer rFVIII and EHL-FVIII products, has the potential to improve replacement therapy in the treatment of hemophilia. The novel therapeutic agents may overcome some of the remaining challenges in current HA treatment and offer the possibility of improving personalized/ individual treatment with better outcomes. Therefore, although nonreplacement therapies and gene therapy appear to be very promising, rFVIII will very likely remain a critical component of the treatment of HA patients because of its physiological mode of action and unique ability to induce tolerance when inhibitors have developed. Even if the exposure to exogenous FVIII could be postponed or at least reduced in the future, it would appear totally premature to consider that replacement therapy with FVIII could be avoided.

\section{Disclosures}

C. Hermans has received honoraria for consulting and speaking fees from Bayer, Shire, Pfizer, Novo Nordisk, Octapharma, Sobi, Roche, CSL Behring and LFB, and research grants from Bayer, Shire, Pfizer, Novo Nordisk, Octapharma, Sobi and CSL Behring. S. Raso states no conflicts of interest.

Writing of this paper was not supported by external funding.

Submitted: March 5, 2018. Revised: April 24, 2018. Accepted: April 25, 2018.

\section{References}

1. Balkaransingh, P., Young, G. Novel therapies and current clinical progress in hemophilia $A$. Ther Adv Hematol 2003, 9(2): 49-61.

2. Srivastava, A., Brewer, A.K., Mauser-Bunschoten, E.P. et al. Guidelines for the management of hemophilia. Haemophilia 2013, 19(1): e1-47.

3. Franchini, M., Lippi, G. Prevention of inhibitor development in hemophilia A in 2016. A glimpse into the future? Thromb Res 2016, 148: 96-100.

4. Tiede, A. Half-life extended factor VIII for the treatment of hemophilia A. J Thromb Haemost 2015, 13: S176-S9.

5. Cafuir, L.A., Kempton, C.L. Current and emerging factor VIII replacement products for hemophilia A. Ther Adv Hematol 2017, 8(10): 303-13.

6. Pool, J.G., Gershgold, E.J., Pappenhagen, A.R. Highpotency antihaemophilic factor concentrate prepared from cryoglobulin. Nature 1964, 203: 312.

7. Gringeri, A. Factor VIII safety: plasma-derived versus recombinant products. Blood Transfus 2011, 9(4): 366-70.

8. Franchini, M. The modern treatment of haemophilia: a narrative review. Blood Transfus 2013, 11(2): 178-82. 
9. Farrugia, A. Evolving perspectives in product safety for haemophilia. Haemophilia 2002, 8: 236-43.

10. Do, H., Healey, J.F., Waller, E.K., Lollar, P. Expression of factor VIII by murine liver sinusoidal endothelial cells. J Biol Chem 1999, 274(28): 19587-92.

11. Jacquemin, M., Neyrinck, A. FVIII production by human lung microvascular endothelial cells. Blood 2006, 108: 515-7.

12. Orlova, N.A., Kovnir, S.V., Vorobiev, I.I., Gabibov, A.G., Vorobiev, A.I. Blood clotting factor VIII: from evolution to therapy. Acta Naturae 2013, 5(2): 19-39.

13. Lenting, P.J., van Mourik, J.A., Mertens, K. The life cycle of coagulation factor VIII in view of its structure and function. Blood 1998, 92(11): 3983-96.

14. Jiang, R., Monroe, T., McRogers, R., Larson, P.J. Manufacturing challenges in the commercial production of recombinant coagulation factor VIII. Haemophilia 2002, 8(Suppl. 2): 1-5.

15. Pipe, S.W. The promise and challenges of bioengineered recombinant clotting factors. J Thromb Haemost 2005, 3(8): 1692-701.

16. Kannicht, C., Ramström, M., Kohla, G., Tiemeyer, M., Casademunt, E., Walter, O., Sandberg, H. Characterisation of the post-translational modifications of a novel, human cell line-derived recombinant human factor VIII. Thromb Res 2013, 131(1): 78-88.

17. Hartmann, J., Croteau, S.E. 2017 clinical trials update: innovations in hemophilia therapy. Am J Hematol 2016, 91(12): 1252-60.

18. Pipe, S.W. Hemophilia: new protein therapeutics. Hematology Am Soc Hematol Educ Program 2010, 2010: 203-9.

19. Afonja, O., Kozak, R., Petraro, P., Michaels, L.A., Mathew, P., Lemm, G., Kessler, C. Baby hamster kidney cell-derived recombinant factor VIII: a quarter century of learning and clinical experience. Expert Rev Hematol 2016, 9(12): 1151-64.

20. Michnick, D.A., Pittman, D.D., Wise, R.J., Kaufman, R.J. Identification of individual tyrosine sulfation sites within factor VIII required for optimal activity and efficient thrombin cleavage. J Biol Chem 1994, 269(31): 20095-102.

21. Franchini, M., Lippi, G. Recombinant factor VIII concentrates. Semin Thromb Hemost 2010, 36(5): 493-7.

22. Santagostino, E. A new recombinant factor VIII: from genetics to clinical use. Drug Des Devel Ther 2014, 8: 2507-15.

23. Miao, H.Z., Sirachainan, N., Palmer, L., Kucab, P., Cunningham, M.A., Kaufman, R.J., Pipe, S.W. Bioengineering of coagulation factor VIR for improved secretion. Blood 2004, 103(9): 3412-9.

24. Di Paola, J., Smith, M.P., Klamroth, R. et al. ReFacto and Advate: a single-dose, randomized, two-period crossover pharmacokinetics study in subjects with haemophilia A. Haemophilia 2007, 13(2): 124-30.

25. Powell, J.S. Recombinant factor VIII in the management of hemophilia A: current use and future promise. Ther Clin Risk Manag 2009, 5(2): 391-402.

26. Ezban, M., Vad, K., Kjalke, M. Turoctocog alfa (NovoEight $($ ) - from design to clinical proof of concept. Eur J Haematol 2014, 93(5): 369-76.

27. Ahmadian, H., Hansen, E.B., Faber, J.H. et al. Molecular design and downstream processing of turoctocog alfa (NovoEight), a B-domain truncated factor VIII molecule. Blood Coagul Fibrinolysis 2016, 27(5): 568-75.

28. Lentz, S.R., Misgav, M., Ozelo, M. et al. Results from a large multinational clinical trial (guardian ${ }^{T M} 1$ ) using prophylactic treatment with turoctocog alfa in adolescent and adult patients with severe haemophilia A: safety and efficacy. Haemophilia 2013, 19: 691-7.

29. Kulkarni, R., Karim, F.A., Glamocanin, S. et al. Results from a large multinational clinical trial (guardian ${ }^{T M} 3$ ) using prophylactic treatment with turoctocog alfa in paediatric patients with severe haemophilia A: safety, efficacy and pharmacokinetics. Haemophilia 2013, 19: 698-705.

30. Ozelo, M.C. Updates from guardian ${ }^{T M}$ : a comprehensive registration programme. Eur J Haematol 2015, 95: 22-9.

31. Lentz, S.R., Cerqueira, M., Janic, D. et al. Interim results from a large multinational extension trial (guardian ${ }^{T M} 2$ ) using turoctocog alfa for prophylaxis and treatment of bleeding in patients with severe haemophilia $A$. Haemophilia 2016, 22(5): e445-9.

32. Saxena, K., Lalezari, S., Oldenburg, J., TseneklidouStoeter, D., Beckmann, H., Yoon, M., Maas Enriquez, M. Efficacy and safety of BAY 81-8973, a full-length recombinant factor VIII: results from the LEOPOLD I trial. Haemophilia 2016, 22: 706-12.

33. Kavakli, K., Yang, R., Rusen, L., Beckmann, H., Tseneklidou-Stoeter, D., Maas Enriquez, M. Prophylaxis vs. on-demand treatment with BAY 81-8973, a fulllength plasma protein-free recombinant factor VIII product: results from a randomized trial (LEOPOLD II). J Thromb Haemost 2015, 13(3): 360-9.

34. Ljung, R., Kenet, G., Mancuso, M.E. et al. BAY 81-8973 safety and efficacy for prophylaxis and treatment of bleeds in previously treated children with severe haemophilia A: results of the LEOPOLD Kids Trial. Haemophilia 2016, 22: 354-60.

35. Shah, A., Delesen, H., Garger, S., Lalezari, S. Pharmacokinetic properties of BAY 81-8973, a full-length recombinant factor VIII. Haemophilia 2015, 21: 766-71.

36. Raso, S., Hermans, C. Lonoctocog alfa (rVIII-SingleChain) for the treatment of haemophilia $A$. Expert Opin Biol Ther 2018, 18(1): 87-94.

37. Schmidbauer, S., Witzel, R., Robbel, L., Sebastian, P., Grammel, N., Metzner, H.J., Schulte, S. Physicochemical 
characterisation of rVIII-SingleChain, a novel recombinant single-chain factor VIII. Thromb Res 2015, 136: 388-95.

38. Zollner, S.B., Raquet, E., Müller-Cohrs, J. et al. Preclinical efficacy and safety of rVIII-SingleChain (CSL627), a novel recombinant single-chain factor VIII. Thromb Res 2013, 132(2): 280-7.

39. Klamroth, R., Simpson, M., von Depka-Prondzinski, M. et al. Comparative pharmacokinetics of rVIII-SingleChain and octocog alfa (Advate $囚)$ in patients with severe haemophilia A. Haemophilia 2016, 22: 730-8.

40. Mahlangu, J., Kuliczkowski, K., Karim, F.A. et al. Efficacy and safety of rVIII-SingleChain: results of a phase 1/3 multicenter clinical trial in severe hemophilia A. Blood 2016, 128(5): 630-7.

41. Casademunt, E., Martinelle, K., Jernberg, M. et al. The first recombinant human coagulation factor VIII of human origin: human cell line and manufacturing characteristics. Eur J Haematol 2012, 89(2): 165-76.

42. Liesner, R.J., Abashidze, M., Aleinikova, O. et al. Immunogenicity, efficacy and safety of Nuwiq ${ }^{\circledR}$ (human- $\mathrm{Cl}$ rhFVIII) in previously untreated patients with severe haemophilia A-Interim results from the NuProtect study. Haemophilia 2018, 24(2): 211-20.

43. Lissitchkov, T., Hampton, K., von Depka, M. et al. Novel, human cell line-derived recombinant factor VIII (humancl rhFVIII; Nuwiq (B) in adults with severe haemophilia A: efficacy and safety. Haemophilia 2016, 22: 225-31.

44. Laffan, M. New products for the treatment of haemophilia. Br J Haematol 2016, 172(1): 23-31.

45. Wynn, T.T., Gumuscu, B. Potential role of a new PEGylated recombinant factor VIII for hemophilia A. J Blood Med 2016, 7: 121-8.

46. Knobe, K., Berntorp, E. New treatments in hemophilia: insights for the clinician. Ther Adv Hematol 2012, 3(3): 165-75.

47. Konkle, B.A., Stasyshyn, O., Chowdary, P. et al. Pegylated, full-length, recombinant factor VIII for prophylactic and on-demand treatment of severe hemophilia A. Blood 2015, 126(9): 1078-85.

48. Stennicke, H.R., Kjalke, M., Karpf, D.M. et al. A novel B-domain O-glycoPEGylated FVIII (N8-GP) demonstrates full efficacy and prolonged effect in hemophilic mice models. Blood 2013, 121(11): 2108-16.

49. Turecek, P.L., Bossard, M.J., Graninger, M. et al. BAX 855, a PEGylated rFVIII product with prolonged half-life. Development, functional and structural characterisation. Hamostaseologie 2012, 32(Suppl. 1): S29-38.

50. Kitchen, S., Kershaw, G., Tiefenbacher, S. Recombinant to modified factor VIII and factor IX - chromogenic and one-stage assays issues. Haemophilia 2016, 22(Suppl.5): 72-7.
51. Coyle, T.E., Reding, M.T., Lin, J.C., Michaels, L.A., Shah, A., Powell, J. Phase I study of BAY 94-9027, a PEGylated $B$-domain-deleted recombinant factor VIII with an extended half-life, in subjects with hemophilia A. J Thromb Haemost 2014, 12(4): 488-96.

52. Reding, M.T., Ng, H.J., Poulsen, L.H. et al. Safety and efficacy of BAY 94-9027, a prolonged-half-life factor VIII. J Thromb Haemost 2017, 15(3): 411-9.

53. Tiede, A., Brand, B., Fischer, R. et al. Enhancing the pharmacokinetic properties of recombinant factor VIII: first-in-human trial of glycoPEGylated recombinant factor VIII in patients with hemophilia A. J Thromb Haemost 2013, 11(4): 670-8.

54. Hampton, K., Chowdary, P., Dunkley, S. et al. First report on the safety and efficacy of an extended half-life glycoPEGylated recombinant FVIII for major surgery in severe haemophilia A. Haemophilia 2017, 23(5): 689-96.

55. Kuo, T.T., Baker, K., Yoshida, M., Qiao, S.W., Aveson, V.G., Lencer, W.I., Blumberg, R.S. Neonatal Fc receptor: from immunity to therapeutics. J Clin Immunol 2010, 30(6): 777-89.

56. Peters, R.T., Toby, G., Lu, Q. et al. Biochemical and functional characterization of a recombinant monomeric factor VIII-Fc fusion protein. J Thromb Haemost 2013, 11(1): 132-41.

57. McCue, J., Kshirsagar, R., Selvitelli, K. et al. Manufacturing process used to produce long-acting recombinant factor VIII FC fusion protein. Biologicals 2015, 43(4): 213-9.

58. Mahlangu, J.N., Ragni, M., Gupta, N. et al. Long-acting recombinant factor VIII FC fusion protein ( $r F V I I I F C$ ) for perioperative haemostatic management in severe haemophilia A. Thromb Haemost 2016, 116(1): 1-8.

59. Mahlangu, J., Powell, J.S., Ragni, M.V. et al. Phase 3 study of recombinant factor VIII FC fusion protein in severe hemophilia A. Blood 2014, 123(3): 317-25.

60. Young, G., Mahlangu, J., Kulkarni, R. et al. Recombinant factor VIII Fc fusion protein for the prevention and treatment of bleeding in children with severe hemophilia A. J Thromb Haemost 2015, 13(6): 967-77.

61. Nolan, B., Mahlangu, J., Perry, D. et al. Long-term safety and efficacy of recombinant factor VIII Fc fusion protein ( $r F V I I F C$ ) in subjects with haemophilia A. Haemophilia 2016, 22(1): 72-80.

62. Pipe, S.W., Montgomery, R.R., Pratt, K.P., Lenting, P.J., Lillicrap, D. Life in the shadow of a dominant partner: the FVIII-VWF association and its clinical implications for hemophilia A. Blood 2016, 128(16): 2007-16.

63. Lillicrap, D., Schiviz, A., Apostol, C. et al. Porcine recombinant factor VIII (Obizur; OBI-1; BAX801): product characteristics and preclinical profile. Haemophilia 2016, 22(2): 308-17. 
64. Kruse-Jarres, R., St-Louis, J., Greist, A. et al. Efficacy and safety of OBI-1, an antihaemophilic factor VIII (recombinant), porcine sequence, in subjects with acquired haemophilia A. Haemophilia 2015, 21(2): 162-70.

65. Mahlangu, J.N., Andreeva, T.A., Macfarlane, D.E., Walsh, C., Key, N.S. Recombinant B-domain-deleted porcine sequence factor VIII (r-pFVIII) for the treatment of bleeding in patients with congenital haemophilia $A$ and inhibitors. Haemophilia 2017, 23(1): 33-41.

66. Hermans, C., Brackmann, H.H., Schinco, P., Auerswald, $\mathrm{G}$. The case for wider use of recombinant factor VIII concentrates. Crit Rev Oncol Hematol 2012, 83(1): 11-20.

67. Wight, J., Paisley, S. The epidemiology of inhibitors in haemophilia A: a systematic review. Haemophilia 2003, 9(4): 418-35.

68. Astermark, J. FVIII inhibitors: pathogenesis and avoidance. Blood 2015, 125(13): 2045-51.

69. Peyvandi, F., Mannucci, P.M., Garagiola, I. et al. A randomized trial of factor VIII and neutralizing antibodies in hemophilia A. N Engl J Med 2016, 374(21): 2054-64.

70. Krishnamoorthy, S., Liu, T., Drager, D. et al. Recombinant factor VIII FC (rFVIIIFC) fusion protein reduces immunogenicity and induces tolerance in hemophilia $A$ mice. Cell Immunol 2016, 301: 30-9.

71. De Groot, A.S., Moise, L., McMurry, J.A. et al. Activation of natural regulatory $T$ cells by IgG Fc-derived peptide "Tregitopes". Blood 2008, 112(8): 3303-11.

72. Peyvandi, F., Garagiola, I., Young, G. The past and future of haemophilia: diagnosis, treatments, and its complications. Lancet 2016, 388(10040): 187-97.

73. Lenting, P.J., Denis, C.V., Christophe, O.D. Emicizumab, a bispecific antibody recognizing coagulation factors
IX and X: how does it actually compare to factor VIII? Blood 2017, 130(23): 2463-8.

74. Franchini, M., Mannucci, P.M. Non-factor replacement therapy for haemophilia: a current update. Blood Transfus 2018: Advanced publication.

75. Prince, R., Bologna, L., Manetti, M. et al. Targeting anticoagulant protein $S$ to improve hemostasis in hemophilia. Blood 2018, 131(12): 1360-71.

76. Lillicrap, D. Bispecific antibody therapy in hemophilia. N Engl J Med 2017, 377(9): 884-5.

77. Oldenburg, J., Levy, G.G. Emicizumab prophylaxis in hemophilia A with inhibitors. N Engl J Med 2017, 377(22): 2194-5.

78. Berntorp, E. Haemophilia treatment in 2030. Haemophilia 2016, 22(Suppl. 5): 15-9.

79. DiMichele, D.M., Hoots, W.K., Pipe, S.W., Rivard, G.E., Santagostino, E. International workshop on immune tolerance induction: consensus recommendations. Haemophilia 2007, 13(Suppl. 1): 1-22.

80. Rode, F., Almholt, K., Petersen, M. et al. Preclinical pharmacokinetics and biodistribution of subcutaneously administered glycoPEGylated recombinant factor VIII (N8-GP) and development of a human pharmacokinetic prediction model. J Thromb Haemost 2018: Advanced publication.

81. Miesbach, W., Meijer, K., Coppens, M. et al. Gene therapy with adeno-associated virus vector 5-human factor IX in adults with hemophilia B. Blood 2018, 131(9): 1022-31.

82. Rangarajan, S., Walsh, L., Lester, W. et al. AAV5-factor VIII gene transfer in severe hemophilia A. N Engl J Med 2017, 377(26): 2519-30. 
